\title{
CONSTRUCTION OF AUTOMORPHIC FORMS AND INTEGRALS
}

\author{
BY \\ DOUGLAS NIEBUR( ${ }^{1}$ )
}

\begin{abstract}
It is well known that modular forms of positive dimension have Fourier coefficients given by certain infinite series involving Kloostermann sums and the modified Bessel function of the first kind. In this paper a functional equation which characterizes all such Fourier series is found. It is also shown that these Fourier series have a construction similar to that of Poincaré series of negative dimension.
\end{abstract}

1. Introduction. In 1936 Hans Rademacher found an infinite series representation for the partition function $p(n)$ [5]. Later he and H. S. Zuckerman found that any modular form of positive dimension has Fourier coefficients which are represented by the Rademacher series in a slightly generalized form [7]. In the opposite direction, Rademacher showed that if one defined a periodic analytic function with a simple pole at $\infty$ and with Fourier coefficients given by the Rademacher series for dimension zero, then this function is an abelian integral for the modular group and its only singularity is the pole at $\infty$. Hence, this function must be, except for a constant term, the modular invariant $J(r)$ [6]. Thus, Rademacher found a new way to define all modular functions. This construction was later generalized by M. I. Knopp to include other discontinuous groups and positive integer dimensions [1]. However, Knopp's construction made use of the notion of "supplementary series" which could not be generalized to nonintegral dimension.

In this paper we show that for certain groups and all positive dimensions, the functions with Fourier coefficients given by the Rademacher series satisfy a certain functional equation; conversely, we show that this functional equation and the principal part at $\infty$ determine the Fourier expansion. This functional equation is analogous to that of an abelian integral, which explains the designation "automorphic integral". The vector space of automorphic integrals contains the space of automorphic forms which are holomorphic in the interior of the upper half-plane, and the difference in the vector space dimensions of the two spaces is related to

Received by the editors July 27, 1973.

AMS (MOS) subject clas sifications (1970). Primary 30A58, $10 \mathrm{D} 15$.

Key words and phrases. Abelian integrals, Fourier coefficients of automorphic forms, Riemann-Roch theorem.

(1) Much of the material in this paper is taken from the author's Ph.D. dissertation submitted to the University of Wisconsin, Madison, in 1968. The author thanks his advisor, Professor Marvin Knopp; for his indulgence. 
the dimension of the space of complementary cusp forms via Petersson's generalized Riemann-Roch theorem [4, p. 194].

2. Definitions of automorphic forms and integrals. Let $\mathcal{G}$ be the group of $2 \times 2$ matrices with real entries and determinant equal to 1 . An element of $\mathcal{G}$ will be denoted by $(a b \mid c d)$. Let $\Gamma$ be a discrete subgroup of $\mathcal{G}$ with a fundamental domain in the upper half-plane $\mathcal{H}$ which has exactly one cusp which we assume to be $\infty$. We also assume $-I=(-10 \mid 0-1) \in \Gamma$.

Throughout this paper $r$ is a fixed positive number and $\chi$ is some multiplier system for $\Gamma$ and dimension $r$; that is, $\chi$ is a function from $\Gamma$ into the unit circle such that, for all $z \in \mathcal{H}$,

$$
\chi\left(M_{3}\right)\left(c_{3} z+d_{3}\right)^{-r}=\chi^{\left(M_{1}\right) \chi\left(M_{2}\right)\left(c_{1} M_{2} z+d_{1}\right)^{-r}\left(c_{2} z+d_{2}\right)^{-r},}
$$

where $M_{j}=\left(* * \mid c_{j} d_{j}\right), 1 \leq j \leq 3$, and $M_{3}=M_{1} M_{2}$. Here, we use the convention that $\log z=\log |z|+i \arg z$, where $\arg z$ is in the interval $[-\pi, \pi)$.

The function $v=\bar{\chi}$ is called the complementary multiplier system for $\chi$; it is a multiplier system for $\Gamma$ and the complementary dimension $-2-r$.

Let $\Gamma_{0}=\{(a b \mid c d) \in \Gamma: c=0\}$. Then $\Gamma_{0}=\left\{ \pm U^{\lambda n}: n \in Z\right\}$, where $U^{\lambda}=$ $(1 \lambda \mid 01)$ for some $\lambda>0$. One easily sees that $\chi\left(U^{\lambda n}\right)=e(n \alpha)$, for some $\alpha$ in the interval $[0,1)$. Here, $e(z)=\exp (2 \pi i z)$. For the complementary multiplier system $v$, we have $v\left(U^{\lambda}\right)=e(\kappa)$, where $\kappa+\alpha=1-[1-\alpha]$.

We now come to the definitions of the relevant function spaces.

Definition. The space of cusp forms on $\Gamma$ for dimension $-2-r$ and multiplier system $v$ is the vector space $\{G\}$ of functions which are holomorphic in $\mathcal{H}$, satisfy $(c z+d)^{-2-r} G(M z)=v(M) G(z)$ for all $M=(* * \mid c d) \in \Gamma$, and have the property that $y^{2+r}|G(x+i y)|^{2}$ is bounded for $x+i y \in \mathcal{H}$. This space is denoted by $\mathcal{C}(-2-r, v)$.

With any $G \in \mathcal{C}(-2-r, v)$ we associate a period function $p$ in the following way: For any $r \in \mathcal{H}, z \in \mathcal{H} \cup\{M \infty: M \in \Gamma\}$, and any vertical path $\gamma$ from $z$ to $i \infty$, put $\Gamma(r+1) p(r, z ; G)=\left[\int_{\gamma} G(w)(w-\bar{r})^{r} d w\right]^{-}$. Here $\Gamma$ denotes the gamma function and - denotes complex conjugation. Since $G$ vanishes exponentially at the cusps, $p$ is holomorphic in $r$ for $r$ confined to some slit plane determined by $z$. Using Cauchy's theorem and the functional equation for $G$, one immediately obtains a functional equation for $p$; i.e.,

$$
v(M)(c r+d)^{r} p(M r, M z ; G)=p(r, z ; G)-p\left(r, M^{-1} \infty ; G\right)
$$

for $M=(* * \mid c d) \in \Gamma$.

Definition. An automorphic integral on $\Gamma$ for dimension $r$ and multiplier system $\chi$ is a function $F$, holomorphic in $\mathcal{H}$, such that, for some $G \in \mathcal{C}(-2-r, v)$, 
(i) $(c r+d)^{r} F(M r)=\chi(M)\left(F(r)-p\left(r, M^{-1} \infty ; G\right)\right)$ for $M=(* * \mid c d) \in \Gamma$, and

(ii) for any cusp $q$ belonging to $\Gamma, F(r)-p(r, q ; G)$ is meromorphic at $q$. In the next section we prove that for each $F$, condition (i) implies $G$ is unique. In order to clarify condition (ii), we note that if $H(r, z)=F(r)-p(r, z ; G)$, then

$$
H(M r, M z)=\chi(M)(c r+d)^{-r} H(r, z)
$$

as a consequence of condition (i) and the previously mentioned functional equation for $p$. Thus, if $\Gamma_{q}$ is the stabilizer of the cusp $q$ in $\Gamma$, then $H(M r, q)=$ $\chi(M)(c r+d)^{-r} H(\tau, q)$ for all $M \in \Gamma_{q}$. It now follows [2, pp. 272-273] that

$$
H(\tau, q)=(\tau-q)^{r} \sum_{n=-\infty}^{\infty} a_{n}(q) e((n+\alpha) A \tau / \lambda),
$$

where $A q=\infty . H(r, q)$ is said to be meromorphic at $q$ and to have order $m+\alpha$ at $q$ if $a_{m}(q) \neq 0$ and $a_{n}(q)=0$ for $n<m$. From equation (2.1) it follows that the orders at $\Gamma$-equivalent cusps are equal. Although it is not necessary for our purposes in this paper, we could define order at any point $z \in \mathcal{H}$ as the order of $H(r, z)$ at $z$, where order at elliptic fixed points must be defined as it is in the theory of automorphic forms.

Definition. The space of automorphic integrals on $\Gamma$ for dimension $r$ and multiplier system $\chi$ which have order at $\infty$ not less than $m$ is denoted by $g(m, r, \chi)$. The subspace of $9(m, r, \chi)$ containing those functions which have an associated cusp form which is identically zero is denoted by $\mathcal{C}(m, r, \chi)$. These functions are called automorphic forms for dimension $r$ and multiplier system $\chi$.

In this paper a basis for the space $g(m, r, \chi)$ will be constructed. To show that this construction yields a spanning set of functions we need one of the following two results:

(a) All automorphic forms of dimension $r$ have Fourier coefficients given by the Rademacher series [2, Chapter IX, and 3].

(b) For sufficiently large $m, \operatorname{dim} \mathcal{C}(-m, r, \chi) \leq m-\operatorname{dim} \mathcal{C}(-2-r, v)$. We shall use the latter result, which is a consequence of Petersson's generalized Riemann-Roch the orem [4, p. 194].

3. A basis for the space of automorphic integrals. A spanning set of automorphic integrals will be constructed in a manner similar to that used to construct cusp forms. We review that construction first.

Let $\nu$ be an integer such that $\nu+\kappa>0$, and let $M=(* * \mid c d) \in \Gamma$. Put

$$
g_{M}(z)=(-2 \pi i(\nu+\kappa) / \lambda)^{r+1} e((\nu+\kappa) M z / \lambda) / \nu(M)(c z+d)^{2+r} \text {. }
$$

Then $g_{M}$ is constant on each right coset of $\Gamma \bmod \Gamma_{0}$ and $(\gamma z+\delta)^{-2-r} g_{M}(L z)=$ 
$v(L) g_{M L}(z)$, if $L=(* * \mid y \delta) \in \Gamma$. Because the sum over all distinct lower rows $(c, d)$ of matrices in $\Gamma$ of $|c z+d|^{-2-r}$ converges uniformly on strips of the form $\{x+i y:|x| \leq A, y \geq \epsilon>0\}$, it follows that the Poincaré series $G_{\nu}(z)=$ $\Sigma_{M \in \Gamma_{0} \backslash \Gamma} g_{M}(z)$ is a cusp form for dimension $-2-r$. The notation in this definition of $G_{\nu}$ indicates that $M$ runs over some set of right coset representatives of $\Gamma \bmod \Gamma_{0}$.

We now try the same construction for dimension $r$.

Definition. Let $\nu$ be an integer such that $\nu+\kappa>0$ and let $M=(* * \mid c d) \in \Gamma$. Let $\mu+\alpha=-\nu-\kappa$ (recall that $\kappa+\alpha$ is 0 or 1 ). Put

$$
s_{M}(\tau)=e((\mu+\alpha) M \tau / \lambda) / \chi(M)\left(c r+d^{\prime}\right)^{-r} \text { and } t_{M}(\tau)=p\left(\tau, M^{-1} \infty, g_{M}\right),
$$

where

$$
\Gamma(r+1) p\left(\tau, M^{-1} \infty ; g_{M}\right)=\left[\int_{M-1 \infty}^{i \infty} 8_{M}(z)(z-\bar{\tau})^{r} d z\right]^{-}
$$

It is clear that $s_{M}(r)$ and $t_{M}(r)$ are holomorphic functions for $r \in \mathcal{H}$ and both are constant on each right coset of $\Gamma \bmod \Gamma_{0}$. It is also easy to see that for $L=(* * \mid \gamma \delta) \in \Gamma$,

$(\gamma \tau+\delta)^{r} s_{M}(L \tau)=\chi(L) s_{M L}(\tau)$ and $(\gamma \tau+\delta)^{r} t_{M}(L \tau)=\chi(L)\left[t_{M L}(\tau)-p\left(\tau, L^{-1} \infty_{\infty} ; g_{M L}\right)\right]$.

In order to define a series analogous to the Poincaré series $G_{\nu}$ we need

Lemma 3.1. Suppose $M=(a * \mid c d) \in \Gamma$ and $c>0$. Tben

$$
s_{M}(\tau)+t_{M}(\tau)=A v(M) e\left((\mu+\alpha) a / \lambda_{c}\right) c^{-1-r}(c \tau+d)^{-1}+O\left(c^{-2-r}|c r+d|^{-2}\right),
$$

where $A=(-2 \pi i(\mu+\alpha) / \lambda)^{r+1} / \Gamma(r+2)$.

Proof. From the definition of $t_{M}$, we have

$$
\begin{aligned}
\Gamma(r+1)(2 \pi i(\nu+\kappa) / \lambda)^{-r-1} t_{M}(\tau) & =\nu(M) \int_{-d / c}^{i \infty} e(-(\nu+\kappa) M \bar{z} / \lambda)(c \bar{z}+d)^{-2-r(\bar{z}-\tau)^{r} d \bar{z}} \\
& =\nu(M)(c \tau+d)^{r} \int_{-i \infty}^{M \infty} e(-(\nu+\kappa) w / \lambda)(w-M \tau)^{r} d w .
\end{aligned}
$$

Here, we have made the substitution $w=M \bar{z}$. The path of integration is now a vertical line in the lower half plane. The simplification of the integrand results from the equation

$$
\left(M^{-1} w-M^{-1} \cdot M \tau\right)^{r}=(w-M \tau)^{r}(-c w+a)^{-r}(-c M r+a)^{-r},
$$

which holds for $c>0, r \in \mathcal{H}$, and $w$ in the lower half plane. We have also used the equations 


$$
v(M) v\left(M^{-1}\right)(-c w+a)^{-2-r}\left(c M^{-1} w+d\right)^{-2-r}=1
$$

and

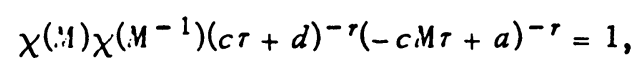

which result from the multiplier system definition. We now make another substitution in the last integral; namely, let $z=(2 \pi i(\nu+\kappa) / \lambda)(w-M \tau)$. Thus,

$$
\Gamma(r+1) t_{M}(\tau)=-v(M)(c \tau+d)^{r} e((\mu+a) M \tau / \lambda) \int_{\beta}^{\infty} e^{-z^{r}} d z
$$

where the integration is a horizontal ray starting at $\beta=2 \pi i(\nu+\kappa) / \lambda c(c r+d)$ and extending to the right.

By solving the first order linear differential equation satisfied by $b(z)=$ $\sum_{j=1}^{\infty} z^{j+r} / \Gamma(j+1+r)$, one finds that $b(z)=e^{z}\left(1-(\Gamma(r+1))^{-1} \int_{z}^{\infty} e^{-t} t^{r} d t\right)$. Thus

$$
s_{M}(\tau)+t_{M}(\tau)=v(M)_{e}((\mu+\alpha) a / c)(-2 \pi i(\mu+\alpha) / c \lambda)^{r} b(\beta) .
$$

The lemma now follows from the simple estimate $b(\beta)=\beta / \Gamma(2+r)+$ $O\left(|\beta|^{2} e^{|\beta|}\right)$.

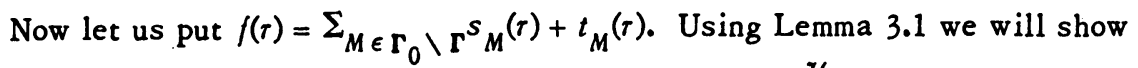
in $\$ 4$ that this sum converges to a function holomorphic in $\mathcal{H}$. The convergence is not absolute; however, we shall show that for a certain family of parallelograms $\left\{P_{N}\right\}, \Sigma_{P_{N}} s_{M}(r)+t_{M}(r)$ converges to $f$ as $N \rightarrow \infty$, where $\Sigma_{P_{N}}$ means a sum over coset representatives with lower row in $P_{N}$. This also holds for any transformed family $\left\{P_{N} L\right\}$, where $L \in \varrho_{\text {and }} P_{N} L=\left\{(c, d) L:(c, d) \in P_{N}\right\}$. With this in mind, it is easy to find the functional equation for $f$ in a formal sense. That is, if $L=(* * \mid \gamma \delta) \in \Gamma$, then

$$
\begin{aligned}
(\gamma \tau+\delta)^{r} f(L \tau) & =\chi(L) \lim _{N \rightarrow \infty} \sum_{P_{N}}\left(s_{M L}(\tau)+t_{M L}(\tau)-p\left(\tau, L^{-1} \infty, g_{M L}\right)\right) \\
& =\chi(L) \lim _{N \rightarrow \infty} \sum_{P_{N} L}\left(s_{M}(\tau)+t_{M}(\tau)-p\left(\tau, L^{-1} \infty, g_{M}\right)\right) \\
& =\chi(L)\left[f(\tau)-p\left(\tau, L^{-1} \infty, G_{\nu}\right)\right] .
\end{aligned}
$$

The major trouble we have in the justification of this formalism comes in the equation $\lim _{N \rightarrow \infty} p\left(r, L^{-1}, \Sigma_{P_{N}} g_{M}\right)=p\left(r, L^{-1}{ }_{\infty}, \lim _{N \rightarrow \infty} \Sigma_{P_{N}} g_{M}\right)$. In fact, this equation is not true if $f$ has a nonzero constant term in its Fourier expansion. In this case the definition of $f$ is altered slightly so that the above functional equation for $f$ remains valid.

Definition. Let $\mu$ be a negative integer and put $\nu=-\mu-\alpha-\kappa$. Define the $\mu$ th Poincaré series $F_{\mu}(r)$ for dimension $r$ as 


$$
1 / 2 a_{0}(\mu+\alpha)+\sum_{c>0} \lim _{N \rightarrow \infty} \sum_{|d| \leq N} s_{M}(\tau)+t_{M}(\tau), \quad M=(* * \mid \dot{c} d) \epsilon \Gamma_{0} \backslash \Gamma
$$

where

$$
a_{0}(\mu)=|2 \pi \mu / \lambda|^{r+1} \frac{2 \pi}{\lambda \Gamma(r+2)} e(r / 4) \sum_{c>0} c^{-2-r} S(\mu ; 0 ; c, \chi),
$$

for $\alpha=0$ and $a_{0}(\mu+\alpha)=0$ for $\alpha>0$. Here, $S$ is the general Kloostermann sum defined by

$$
S(m, n ; c, \chi)=\sum_{M} \bar{\chi}(M) e\left((a(m+\alpha)+d(n+\alpha)) / \lambda_{c}\right),
$$

where $M$ runs over the finite set $\{(a * \mid c d) \in \Gamma: 0 \leq a<\lambda|c|, 0 \leq d<\lambda|c|\}$.

In $\$ 4$ we show that $F_{\mu}$ is holomorphic in $\mathcal{H}$, and at the same time we find the Rademacher formula for the Fourier coefficients. We then prove the

Main Theorem. $F_{\mu}(\tau)$ is an automorphic integral with principal part $e((\mu+\alpha) \tau / \lambda)$ at $\infty$, and its associated cusp form is $G_{\nu}$.

Assuming this result for now, we show that $\left\{F_{\mu}:-m \leq \mu \leq-1\right\}$ is a basis for $9(-m, r, \chi)$.

Lemma 3.2. Let $G \in \mathcal{C}(-2-r, v)$. Then $p(r, M \infty ; G)=0$ for all $M \in \Gamma$ if and only if $G$ is identically zero. Hence, a unique cusp form is associated with eacb automorphic integral.

Proof. Suppose first that $r$ is an integer and $p(\tau, M ; G)=0$ for all $M \in \Gamma$. Let

$$
r ! F(\tau)=\int_{\tau}^{i \infty} G(z)(z-\tau)^{r} d z=\int_{0}^{i \infty} G(z+\tau) z^{r} d z .
$$

Then $F(\tau)$ is holomorphic for $\tau \in \mathcal{H}$, and if $M=(* * \mid c d) \in \Gamma$, then

$$
\begin{aligned}
(c \tau+d)^{\tau} F(M \tau) & =\frac{1}{\tau !} \int_{\tau}^{M-1 \infty} G(M z)(c z+d)^{-2-\tau}(z-\tau) d z \\
& =v(M)\left[F(\tau)-p\left(\tau, M^{-1} \infty ; G\right)\right]=v(M) F(\tau) .
\end{aligned}
$$

If $G(z)=\Sigma_{m+\kappa>0 b_{m}} e((m+\kappa) z / \lambda)$, then

$$
F(\tau)=(i \lambda / 2 \pi)^{r+1} \sum_{m+\kappa>0}(m+\kappa)^{-r-1} b_{m} e((m+\kappa) r / \lambda) .
$$

Thus $F$ is a cusp form of positive dimension, which implies $b_{m}=0$ for all $m$. This proves the lemma if $r$ is an integer.

Now suppose $r$ is not an integer and $p(r, M \infty ; G)=\left[p\left(\bar{r}, M_{\infty} ; G\right)\right]^{-}$for some $M \in \Gamma$ and $M \notin \Gamma_{0}$, where $\operatorname{Re} r \not M \infty$. For $t>0$ let $g(t)=G\left(M_{\infty}+i t\right), g(0)=0$, 
and $g(-t)=\bar{g}(t)$. Then $g$ is continuous and vanishes exponentially at $\pm \infty$. Put

$$
f(y)=\int_{-\infty}^{\infty} g(t)(y-t+i)^{r} d t=e((r+1) / 4) \Gamma(r+1)\left[p(r, M \infty, G)-\bar{p}\left(\bar{r}, M_{\infty}, G\right)\right],
$$

where $\tau=M_{\infty}-1+i y$. By hypothesis, $f(y)=0$ for all $y$. Differentiating the integral expression $[r]+2$ times, we find that $(g * k)(y)=\int_{-\infty}^{\infty} g(t) k(y-t) d t$ is zero for all $y$, where $k(t)=(t+i)^{\beta}$ and $\beta=r-[r]-2$. Since the Fourier transform of $k$ does not vanish on a nonempty open interval and the transform of $g$ is analytic, it follows that $g=0$. Thus $G$ is zero.

Theorem 3.3. If $m \geq 1,\left\{F_{\mu}:-m \leq \mu \leq-1\right\}$ is a basis for $g(-m, r, \chi)$. If $m \leq 0, g(-m, r, \chi)=\{0\}$.

Proof. Let $m \geq 1$. Since $F_{\mu}(\tau)$ has principal part $e((\mu+\alpha) \tau / \lambda)$ at $\infty$, the functions are clearly linearly independent. Hence, $\operatorname{dim} g(-m, r, \chi) \geq m$. On the other hand, Lemma 3.2 implies that $\operatorname{dim} 9(-m, r, \chi)-\operatorname{dim} \mathcal{C}(-m, r, \chi) \leq$ $\operatorname{dim} \mathcal{C}(-2-r, v)$. But statement (b) at the end of $\$ 2$ then implies that there is an $M>0$ such that $\operatorname{dim} 9(-m, r, \chi) \leq m$ for all $m \geq M$. This proves the theorem for all $m \geq M$. But for all $m<M, g(-m, r, \chi) \subset g(-M, r, \chi)$, from which it follows that $\left\{F_{\mu}:-m \leq \mu \leq-1\right\}$ spans $\mathscr{G}(-m, r, \chi)$; hence $\mathscr{G}(0, r, \chi)=\{0\}$.

4. Proof of the Main Theorem. The idea behind the proof is the conversion of partial fraction expansions to Fourier expansions via the Lipschitz summation formula, which is a special case of the Poisson summation formula. The necessary formula is given in

Lemma 4.1. Let $0 \leq a<1, \beta=[1-\alpha]+\alpha, p>0, N$ be a positive integer, and $Q=N+1 / 2$. If $\operatorname{Re} w>0$ and $|\operatorname{Im} w|<Q$, then

$$
\sum_{n=-N}^{N} e(n \alpha)(w+i n)^{-p}=\frac{(2 \pi)^{p}}{\Gamma(p)} \sum_{m+a>0}(m+\alpha)^{p-1} e(i(m+\alpha) w)+E,
$$

where the error $E=E(w, p, Q)$ is $i Q^{1-p} \int_{-\infty}^{\infty}(b(x-i)-b(x+i))(1+\exp (2 \pi x Q))^{-1} d x$ and $h(t)=(t+w / Q)^{-p} \exp (2 \pi t Q \beta)$.

Proof. Without loss of generality we assume $w$ is positive. Let $f(z)=$ $2 e(-i(z-w) \beta) / z^{p}(e(-i(z-w))-1)$. Then $f$ has a pole at $w+i n$ for each integer $n$ and the residue is $e(n \alpha)(w+i n)^{-p}$. Let $\gamma$ be a boundary path for the rectangle with corners $\pm M \pm i Q$ which is slit along the negative axis; apply the residue theorem and let $M \rightarrow \infty$. The integrals along the horizontal lines give the error $E$. The main term comes from the infinite loop integral; i.e., we insert the power series for $(e(-i(z-w))-1)^{-1}$ and integrate term-by-term, using the Hankel loop integral for $1 / \Gamma(p)$. 
Corollary (Lipschitz summation formula). If $\operatorname{Re} w>0, p \geq 1$, and $0 \leq a<1$, then

$$
\begin{aligned}
\lim _{N \rightarrow \infty} \sum_{n=-N}^{N} e(n a)(w+i n)^{-p}-\frac{(2 \pi)^{p}}{\Gamma(p)} \sum_{m+a>0}(m+\alpha)^{p-1} e(i(m+\alpha) w) & \\
& = \begin{cases}\pi & \text { if } a=0 \text { and } p=1 \\
0 & \text { otberwise. }\end{cases}
\end{aligned}
$$

Proof. If $p=1$ and $\alpha=0$ then as $Q \rightarrow \infty, E(w, p, Q) \rightarrow 2 \int_{0}^{\infty}\left(x^{2}+1\right)^{-1} d x=$ $\pi$. If $\alpha>0$, then $0<\beta<1$ and the error tends to zero. If $a=0$, the integral defining. $E$ is bounded; hence, $E \rightarrow 0$ as $Q \rightarrow \infty$ if $p>1$.

For the remainder of $\$ 4, \mu$ is a fixed negative integer and $\nu=-\mu-\alpha-\kappa$. A sum over a set of right coset representatives for $\Gamma \bmod \Gamma_{0}$ will be written as a sum over $I \cup\{(a * \mid c d+n \lambda c)$ : $c>0,0 \leq a<c \lambda, 0 \leq d \leq c \lambda, n \in \mathbf{Z}\}$. Further, a sum over this last set is represented by $\bar{\Sigma}_{c>0} \Sigma_{d}$, or by $\Sigma_{c>0} \Sigma_{d}^{*} \Sigma_{n}$.

Lemma 4.2. If $F_{\mu}(\tau)=1 / 2 a_{0}(\mu+\alpha)+\Sigma_{c>0} \lim _{N \rightarrow \infty} \Sigma_{|d| \leq N} s_{M}(\tau)+t_{M}(\tau)$, then $F_{\mu}(\tau)$ is bolomorphic for $\tau \in \mathcal{H}$ and $F_{\mu}(\tau)=e((\mu+\alpha) \tau / \lambda)+$ $\Sigma_{m=0}^{\infty} a_{m}(\mu+\alpha) e((m+\alpha) \tau / \lambda)$, where

$$
\begin{aligned}
a_{m}(\mu+\alpha)= & (2 \pi / \lambda) e(r / 4)|(\mu+\alpha) /(m+\alpha)|^{(r+1) / 2} \sum_{c>0} c^{-1} S(\mu, m ; c, \chi) \\
& \cdot I_{r+1}\left(4 \pi|(\mu+\alpha)(m+\alpha)|^{1 / 2} / \lambda c\right) .
\end{aligned}
$$

Here, $a_{0}$ and $S$ are defined by (3.4) and (3.5), and $I_{r+1}$ is the modified Bessel function of the first kind.

Proof. Let $c>0$, and put $m_{0}=|m+\alpha| / \lambda$ for $m \in \mathbf{Z}$. From equation (3.2) of Lemma 3.1, we obtain

$$
\begin{aligned}
\sum_{|d| \leq N} s_{M}(\tau)+t_{M}(\tau)= & \sum_{d}^{*} v(M) e\left(-\mu_{0} a / c\right)\left(2 \pi i \mu_{0} / c\right)^{r} \\
& \cdot \sum_{|n| \leq N / c \lambda} e(n \alpha) \sum_{j=1}^{\infty} \frac{\left(2 \pi i \mu_{0} / c(c r+d-n c \lambda)\right)^{j}}{\Gamma(j+1+r)}+O\left(N^{-1}\right) .
\end{aligned}
$$

The term $O\left(N^{-1}\right)$ accounts for the small difference in the ranges of summation; i.e., the terms for $d=d^{*} \pm N$. We now interchange the sums on $n$ and $j$ and let $N \rightarrow \infty$. Note that the double sum over $\{(j, n): 2 \leq j, n \in \mathbb{Z}\}$ converges absolutely. Using the Lipschitz summation formula, we obtain

$$
\lim _{N \rightarrow \infty} \sum_{|d| \leq N} s_{M}(\tau)+t_{M}(\tau)=\sum_{d}^{*} v(M) e\left(-\mu_{0} a / c\right)\left(2 \pi i \mu_{0} / c\right)^{r} \Phi,
$$


where

$$
\Phi=\frac{2[1-\alpha] \pi^{2} \mu_{0}}{c^{2} \lambda \Gamma(2+r)}+\sum_{j=1}^{\infty} \frac{\left(4 \pi^{2} \mu_{0} / c^{2} \lambda\right)^{j}}{\Gamma(j+r+1) \Gamma(j)} \sum_{m 0>0}(m+\alpha)^{j-1} e\left(m_{0}(\tau+d / c)\right) .
$$

Interchanging the double sum defining $\Phi$, we finally have

$$
\begin{aligned}
\lim _{N \rightarrow \infty} & \sum_{|d| S N} s_{M}(\tau)+t_{M}(\tau) \\
= & \frac{[1-a] e(r / 4)\left|2 \pi \mu / \lambda_{c}\right|^{r+1}}{c \lambda \Gamma(r+2)} \pi S(\mu, 0 ; c, \chi) \\
& +\frac{2 \pi e(r / 4)}{c \lambda} \sum_{m 0>0} e\left(m_{0} \tau\right) S(\mu, m ; c, \chi)\left|\mu_{0} / m_{0}\right|^{(r+1) / 2} I_{r+1}\left(4 \pi\left|\mu_{0} m_{0}\right|^{1 / 2} / c\right)
\end{aligned}
$$

We now sum both sides of equation (4.2) over all $c>0$ and add the term $s_{I}(\tau)+$ $t_{l}(\tau)=e\left(-\mu_{0} \tau\right)$ to the result. This proves $F_{\mu}$ has the stated Fourier expansion. Using simple estimates, one sees that this Fourier series converges absolutely for $\tau \in \mathcal{H}[1]$.

We now show that $F_{\mu}$ can be defined as the limit of finite double sums.

Lemma 4.3. Let $F_{\mu}$ be defined as in Lemma 4.2. Suppose that $L \in \mathcal{G}$ and for eacb integer $N, P_{N} L=\left\{(x, y) L:|x| \leq N,|y| \leq N^{2}\right\}$. If $r \in \mathcal{H}$, then $F_{\mu}(\tau)=$ $1 / 2 a_{0}(\mu+\alpha)+\lim _{N \rightarrow \infty} \Sigma_{M \in A}{ }^{L} s_{M}(\tau)+t_{M}(\tau)$, where the index set is $A_{N} L=$ $I \cup\left\{M=(* * \mid c d) \in \Gamma_{0} \backslash \Gamma: c>0,(c, d) \in P_{N} L\right\}$.

Proof. From Lemma 3.1, $s_{M}(\tau)+t_{M}(\tau)=A v(M) e\left(-\mu_{0} a / c\right) / c^{1+r}(c r+d)+$ $R_{M}(r)$, where $\mu_{0}=|\mu+\alpha| / \lambda, A$ is independent of $M=(a * \mid c d)$, and $R_{M}(r)=$

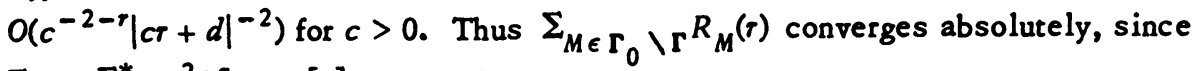
$\Sigma_{c>0} \Sigma_{d}^{*} c^{-2-r}<\infty[1]$. Now, if

$$
T=\sum_{c>0} \lim _{N \rightarrow \infty} \sum_{|d| \leq N} \frac{v(M) e\left(-\mu_{0} a / c\right)}{c^{1+r}(c r+d)} \quad \text { and } T_{N}(L)=\sum_{M \in A_{N} L} \frac{v(M) e\left(-\mu_{0} a / c\right)}{c^{1+r}(c r+d)}
$$

then we must show $\lim _{N \rightarrow \infty} T_{N}(L)=T$. Using the Lipschitz summation formula, we see that

$$
T=\sum_{c>0} c^{-2-r} \sum_{d}^{*} v(M) e\left(-\mu_{0} a / c\right)(-\pi i / \lambda)\left\{[1-a]+2 \sum_{m} e\left(m_{0}(r+d / c)\right)\right\},
$$

where $\Sigma_{m}$ is a sum over integers $m$ such that $\lambda m_{0}=m+a>0$.

Suppose, first, that $L=\left(\rho \sigma \mid 0 \rho^{-1}\right)$ and $\rho>0$. If $w=-i(r+d / c) / \lambda$, then 


$$
T_{N}(L)=\sum_{0<c \leq N \rho} c^{-2-r} \sum_{d}^{*} v(M) e\left(-\mu_{0} a / c\right)(-i / \lambda) \sum_{n} e(n \alpha)(w+i n)^{-1},
$$

where $n$ ranges over the integers such that $|d-\sigma c / \rho-n c \lambda| \leq N^{2} / \rho$. We may replace this range by $|n| \leq N^{2} / \rho c \lambda$ and the error is $O\left(\rho c \lambda / N^{2}\right)$. Then by Lemma 4.1 , the resulting sum on $n$ is $2 \pi \Sigma_{m} e\left(m_{0}(\tau+d / c)\right)+E(w, 1, Q)$, where $Q=1 / 2+$ $\left[N^{2} / \rho c \lambda\right]$. Since $0<c \leq N \rho$ in the sum defining $T_{N}(L)$, and $E(w, 1, Q) \rightarrow$ $\pi[1-\alpha]$ as $Q \rightarrow \infty$, it follows that $T_{N}(L) \rightarrow T$ as $N \rightarrow \infty$.

Finally, suppose that $L=(* * \mid \gamma \delta) \in \varrho_{\text {and }} \gamma>0$. The boundary of $P_{N} L$ intersects the vertical axis of the Cartesian plane at $\pm N / \gamma$; hence, $T_{N}(L)=$ $T_{K}\left(L^{\prime}\right)+R_{K}$, where $L^{\prime}=\left(\gamma 0 \mid 0 \gamma^{-1}\right), K=N^{1 / 2}$, and $\left|R_{K}\right| \leq$ $\Sigma_{c} \Sigma_{d}^{*} \Sigma_{n} c^{-1-r}|c r+d-n c \lambda|^{-1}$. In this majorizing sum, $c$ and $n$ are restricted by the conditions (1) $0<c \leq \gamma K$ and $\delta^{2} c / \lambda \geq \delta\left(d-n c \lambda \pm K^{2} / \gamma\right) \geq 0$ or (2) $\gamma K<c$ and $|d-n c \lambda| \leq\left(|\delta| c+K^{2}\right) / \gamma$. Thus,

$$
R_{K} \ll \sum_{0<c \leq \gamma K} \sum_{d}^{*} c^{-2-r} \log \left(1+c \lambda K^{-1}\right)+\sum_{\gamma K<c d} \sum_{d}^{*} c^{-2-r} \log K,
$$

and $R_{K} \rightarrow 0$ as $K \rightarrow \infty$, since $\Sigma_{c>x} \Sigma_{d}^{*} c^{-2-r} \ll x^{-r}$. Hence $\lim _{N \rightarrow \infty} T_{N}(L)=$ $\lim _{K \rightarrow \infty} T_{K}\left(L^{\prime}\right)=T$.

The problem of integrating the Poincaré series term-by-term is now solved by

Lemma 4.4. Let $L \in \mathcal{G}$ and define $P_{N} L$ and $A_{N} L$ as in Lemma 4.3. Let $G_{N}(z ; L)=\Sigma_{M \in A_{N} L} g_{M}(z)$ and $G(z)=\lim _{N \rightarrow \infty} G_{N}(z ; L)$. Then for any $\tau, \omega \in \mathcal{H}$, $\lim _{N \rightarrow \infty} p\left(r, \omega ; G_{N}\right)=p(r, \omega, G)-1 / 2 a_{0}(\mu+\alpha)$.

Proof. Assume that $L=\left(\rho \sigma \mid 0 \rho^{-1}\right)$ with $\rho>0$. Let $m_{0}=(m+\kappa) / \lambda$ for any integer $m$. Then

$$
\begin{aligned}
& \left(-2 \pi i \nu_{0}\right)^{-r-1} G_{N}(z ; L)=\sum_{M \in A_{N} L} e^{\left(\nu_{0}, H z\right) \chi(M)(c z+d)^{-2-r}} \\
& =e\left(\nu_{0} z\right)+\sum_{0<c \leq N \rho} \sum_{d}^{*} \chi^{(M) e\left(\nu_{0} a / c\right)} \sum_{n} e\left(n \kappa-\nu_{0} / d(c z+d-n c \lambda)\right)(c z+d-n c \lambda)^{-2-r},
\end{aligned}
$$

where $n$ ranges over the integers such that $|d-\sigma c / \rho-n c \lambda| \leq N^{2} / \rho$. The limits on $n$ are then changed to $|n| \leq N^{2} / \rho c \lambda$ and the error is $O\left(\left(|c z|+N^{2} / \rho \lambda\right)^{-2-r}\right)$. The power series for $\exp \left(-2 \pi i \nu_{0} / c(c z+d-n c \lambda)\right)$ is now inserted and interchanged with the sum on $N$, and then Lemma 4.1 is applied. Let $w=-i(z+d / c)$ and $Q=1 / 2+\left[N^{2} / \rho c \lambda\right]$. We obtain

$$
\left(-2 \pi i \nu_{0}\right)^{-r-1} G_{N}(z ; L)=e\left(\nu_{0} z\right)+\Phi_{1}+\Phi_{2}+O\left((|z|+N)^{-2-r}\right),
$$

where 


$$
\begin{aligned}
& \Phi_{l}=-e(-r / 4) \sum_{0<c \leq \rho N}(c \lambda)^{-2-r} \sum_{d}^{*} \chi(M) e\left(\nu_{0} a / c\right) \sum_{j=0}^{\infty}\left(-2 \pi \nu_{0} / c^{2} \lambda\right)^{j} \phi_{l} / j !, \\
& \phi_{1}=\frac{(2 \pi)^{2+j+r}}{\Gamma(2+j+r)} \sum_{m 0>0}\left(\lambda_{m_{0}}\right)^{1+j+r} e\left(i w m_{0}\right), \text { and } \phi_{2}=E(w, 2+j+r, Q)
\end{aligned}
$$

as defined in Lemma 4.1. Using the Lipschitz formula on $\phi_{1}$ and then backtracking to the beginning of the proof, we find that $\Phi_{1}=\Sigma_{0<c \leq N \rho} \Sigma_{d} g_{M}(z)$. Because the inner sum is over all $d$ such that $(* * \mid c d) \in \Gamma_{0} \backslash \Gamma, \Phi_{1}$ has the Fourier expansion $\Sigma_{m_{0}>0 c_{m}}(N) e\left(m_{0} z\right)$, and $\lim _{N \rightarrow \infty} c_{m}(N)=c_{m}$, where $\left(-2 \pi i \nu_{0}\right)^{-r-1} G(z)=e\left(\nu_{0} z\right)+\Sigma_{m_{0}}>0 c_{m} e\left(m_{0} z\right)$. Hence, the dominated convergence theorem can be applied to $\lim _{N \rightarrow \infty} p\left(r, \omega ; \Phi_{1}\right)$.

To complete the proof of the case $L=\left(\rho \sigma \mid 0 \rho^{-1}\right)$ it is now sufficient to show

$$
\lim _{N \rightarrow \infty} p\left(r, \omega ; \Phi_{2}\right)=-\left(2 \pi i \nu_{0}\right)^{-r-1} a_{0}(\mu+\alpha) .
$$

Let $\omega=X+i Y$, and as a path of integration for the integral defining $p$, take $z=X+i Q \lambda y$, where $Y / Q \lambda=\epsilon \leq y<\infty$. Thus,

$$
\begin{aligned}
\lambda \Gamma(r+1)\left[p\left(r, \omega, \Phi_{2}\right)\right]^{-} & =\lambda \int_{\omega}^{i \infty} \cdot \Phi_{2}(z-\bar{\tau})^{r} d z \\
& =\sum_{0<c \leq N \rho} \sum_{d}^{*} c^{-2-r} \chi(M) e\left(\nu_{0} a / c\right) B(c, d, N),
\end{aligned}
$$

where

$$
B(c, d, N)=\int_{\epsilon}^{\infty} \int_{-\infty}^{\infty}(y-i x)^{r}(b(t-i, y)-b(t+i, y))(1+e(-i t Q))^{-1} d t d y
$$

and $b(t, y)=e\left(-i t Q \beta+i \nu_{0} / c^{2} \lambda Q(t+y+b)\right)(t+y+b)^{-2-r}$. Here, $x=$ $(X-\bar{\tau}) / Q \lambda, b=-i(X+d / c) / Q \lambda, Q=1 / 2+\left[N^{2} / \rho c \lambda\right]$, and $\beta=[1-\kappa]+\kappa$.

We now show that $B(c, d, N)$ converges uniformly in $c$ and $d$ to $-i \pi[1-\kappa] \Gamma(1+r) / \Gamma(2+r)$, if $0<c \leq N \rho$ and $0 \leq d<c \lambda$. Note that under these conditions, $x, b$, and $\epsilon$ are uniformly bounded by a multiple of $N^{-1}$.

If $\kappa>0$, the proof is easy. For then, $\delta=\min \{\beta, 1-\beta\}>0$ and

$$
\int_{\epsilon}^{\infty}|y-i x|^{r}|b(t-i, y)-b(t+i, y)||1-e(-i t Q)|^{-1} d y \ll e^{-\delta Q|t|},
$$

uniformly in $c$ and $d$. Hence, $B(c, d, N) \rightarrow 0$ uniformly as $N \rightarrow \infty$.

Now suppose $\kappa=0$ and $\beta=1$. Then

$$
b(t \pm i)=-\exp (2 \pi Q t)\left((t+y \pm i)^{-2-r}+O\left(N^{-1}|t+y+i|^{-3-r}\right)\right)
$$


and

$$
(y-i x)^{r}=y^{r}+O\left(N^{-1} y^{r-1}\right) \text { for } y \geq 2|x| .
$$

Thus Fubini's theorem is applicable and

$$
\begin{aligned}
\int_{\epsilon}^{\infty}(y-i x)^{r}(b(t-i, y)-b(t+i, y)) d y= & \int_{0}^{\infty} y^{r}\left[(t+y+i)^{-2-r}-(t+y-i)^{-2-r}\right] d y \\
& +O\left(N^{-1}|t+i|^{-2}\right) .
\end{aligned}
$$

But $\int_{0}^{\infty} y^{r}(t+y \pm i)^{-2-r} d y=(t \pm i)^{-1} \Gamma(1+r) / \Gamma(2+r)$. Thus, $B(c, d, N)$ converges uniformly in $c$ and $d$ to $-i \pi \Gamma(1+r) / \Gamma(2+r)$. Hence,

$$
\begin{aligned}
\lim _{N \rightarrow \infty}\left[p\left(r, \omega, \Phi_{2}\right)\right]^{-} & =\frac{-i \pi}{\lambda \Gamma(2+r)} \cdot \sum_{c>0} c^{-2-r}[S(\mu, 0 ; c, \chi)]^{-} \\
& =-1 / 2\left(-2 \pi i \nu_{0}\right)^{-r-1}\left[a_{0}(\mu+\alpha)\right]^{-} .
\end{aligned}
$$

This proves the lemma in case $L=\left(\rho \sigma \mid 0 \rho^{-1}\right)$.

In case $L=(* * \mid \gamma \delta) \in \mathcal{G}, \gamma>0$, we use the argument in the proof of Lemma 4.3 to reduce this to the previous case. That is, $G_{N}(z ; L)=G_{K}\left(z ; L^{\prime}\right)+R_{K}(z)$, where $L^{\prime}=\left(y 0 \mid 0 \gamma^{-1}\right), K=N^{1 / 2}$, and $\left|R_{K}(z)\right| \leq \Sigma_{c} \Sigma_{d}|c z+d|^{-2-r}$. Again, this majorizing sum is restricted to those $c$ and $d$ satisfying (1) $0<c \leq \gamma K$ and $\delta^{2} c / \gamma \geq \delta\left(d \pm K^{2} / \gamma\right) \geq 0$, or (2) $\gamma K<c$ and $|d| \leq\left(|\delta| c+K^{2}\right) / \gamma$. Since $\int_{\omega}^{i \infty}|c z+d|^{-2-r}|z-\bar{\tau}|^{r} d z \ll c^{-1-r}|c \omega+d|^{-1}$, we have $\lim _{K \rightarrow \infty} p\left(r, \omega, R_{K}\right)=0$ for the same reasons that were given in the proof of Lemma 4.3.

Corollary 4.5. Let $L \in \oint$ and define $G_{N}(z ; L)$ and $G$ as in Lemma 4.4. Then for any $M=(* * \mid c d) \in \Gamma$,

$$
\lim _{N \rightarrow \infty} \rho\left(r, M^{-1}{ }_{\infty} ; G_{N}\right)=p\left(r, M^{-1} \infty ; G\right)+1 / 2 a_{0}(\mu+\alpha)\left(\nu(M)(c r+d)^{r}-1\right) .
$$

Proof. If $c=0$ then both sides of the equation are zero. Assume, then, that $M=(a * \mid c d)$ and $c \neq 0$. Let $\omega=M^{-1} \infty+i$ and $\Gamma(r+1) I_{N}(r)=$ $\int_{M^{-1}}^{\omega} G_{N}(z ; L)(z-\bar{\tau})^{r} d z$. Replacing $z$ by $M^{-1} z$ in this integral, we obtain

$$
\Gamma(r+1) I_{N}(\tau)=-\nu\left(M^{-1}\right)(-c M \bar{r}+a)^{-r} \int_{M \omega}^{i \infty} G_{N}\left(z ; L M^{-1}\right)(z-M \bar{r})^{r} d z .
$$

Hence,

$$
\lim _{N \rightarrow \infty}\left[I_{N}(\tau)\right]^{-}=-\chi(M-1)(-c M r+a)^{-r}\left(p(M r, M \omega ; G)-1 / 2 a_{0}(\mu+a)\right)
$$

by Lemma 4.4. From the multiplier condition we have $\chi\left(M^{-1}\right)(-c M r+a)^{-r}=$ $v(M)(c r+d)^{r}$. Thus, we finally obtain the equation 


$$
\lim _{N \rightarrow \infty}\left[I_{N}(\tau)\right]^{-}=p\left(\tau, M^{-1} ; G\right)-p(\tau, \omega ; G)+1 / 2 a_{0}(\mu+a) v(M)(c \tau+d)^{r} .
$$

On the other hand, Lemma 4.4 shows directly that

$$
\lim _{N \rightarrow \infty} p\left(\tau, M^{-1} \infty, G_{N}\right)-\left[I_{N}(\tau)\right]^{-}=p(\tau, \omega, G)-1 / 2 a_{0}(\mu+\alpha) .
$$

With all this analysis finished, the proof of the main theorem is reduced to formalities. Lemma 4.2 shows that $F_{\mu}(\tau)$ is holomorphic for $\tau \in \mathcal{H}$ and it has principal part $e((\mu+\alpha) \tau / \lambda)$ at $\infty$. If $L=(* * \mid c d) \in \Gamma$, then Lemma 4.3 implies $F_{\mu}(\tau)=1 / 2 a_{0}(\mu+\alpha)+\lim _{N \rightarrow \infty} \Sigma{ }_{M \in A_{N} L^{-1} s_{M}(\tau)+t_{M}(\tau) \text {. The functional equations }}$
for $s_{M}$ and $t_{M}$ imply that

$$
\begin{aligned}
v(L)(\gamma \tau+\delta)^{r} F_{\mu}(L \tau)= & 1 / 2 v(L)(\gamma \tau+\delta)^{r} a_{0}(\mu+\alpha) \\
& +\lim _{N \rightarrow \infty} \sum_{M \in A_{N} I} s_{M}(\tau)+t_{M}(\tau)-p\left(\tau, L^{-1} \infty, g_{M}\right) .
\end{aligned}
$$

Finally, Corollary 4.5 shows that the right-hand side of this equation is $1 / 2 a_{0}(\mu+\alpha)+\lim _{N \rightarrow \infty} \Sigma_{M \in A} I^{s} s^{(\tau)}+t_{M}(\tau)-p\left(\tau, L^{-1} \infty, G_{\nu}\right)$. Hence, Lemma 4.3 implies that $F_{\mu}$ satisfies the functional equation of an automorphic integral with associated cusp form $G_{\nu^{\circ}}$.

\section{BIBLIOGRAPHY}

1. M. I. Knopp, Construction of automorphic forms on H-groups and supplementary Fourier series, Trans. Amer. Math. Soc. 103 (1962), 168-188. MR 25 \#197.

2. Joseph Lehner, Discontinuous groups and automorphic functions, Math. Surveys, no. 8, Amer. Math. Soc., Providence, R. I., 1964. MR 29 \#1332.

3. Hans Petersson, Konstruktion der Modulformen und der zu gewissen Grenzkreisgruppen gehörigen automorphen Formen von positiver reeller Dimension und die vollständige Bestimmung ihrer Fourierkoeffizienten, S.-B. Heidelberger Akad. Wiss. Math.Nat. K1. 1950, 417-494. MR 12,806.

4. - Zur analytischen Theorie der Grenzkreisgruppen. II, Math. Ann. 115 (1938), 175-204.

5. Hans Rademacher, On the partition function $p(n)$, Proc. London Math. Soc. (2) 43 (1937), 241-254.

6. - The Fourier series and the functional equation of the absolute modular invariant $J(\tau)$, Amer. J. Math. 61 (1939), 237-248.

7. Hans Rademacher and H. S. Zuckerman, On the Fourier coefficients of certain modular forms of positive dimension, Ann. of Math. 39 (1938), 433-462.

DEPARTMENT OF MATHEMATICS, UNIVERSITY OF MARYLAND, COLLEGE PARK, MARY• LAND 20742 